\title{
Upgraded LHC experiments as a check of non-perturbative ef- fects of the Electro-Weak Interaction
}

\author{
Boris A. Arbuzov ${ }^{1, \star}$ and Ivan V. Zaitsev ${ }^{1, \star \star}$ \\ ${ }^{1}$ M.V. Lomonosov Moscow State University
}

\begin{abstract}
Recently reported diphoton excesse at LHC is interpreted to be connected with heavy $W W$ zero spin resonances. The resonances appears due to the wouldbe anomalous triple interaction of the weak bosons, which is defined by coupling constant $\lambda$. The $\gamma \gamma 750 \mathrm{GeV}$ anomaly is considered to correspond to weak isotopic spin 0 pseudoscalar state. We obtain estimates for the effect, which qualitatively agree with ATLAS data. Effects are predicted in a production of $W^{+} W^{-},(Z, \gamma)(Z, \gamma)$ via resonance $X_{P S}$ with $M_{P S} \simeq$ $750 \mathrm{GeV}$, which could be reliably checked at the upgraded LHC at $\sqrt{s}=13 \mathrm{TeV}$. In coupling constant of the triple anomalous interaction is estimated to be $\lambda=-0.010 \pm$ 0.005 in an agreement with existing restrictions. Specific predictions of the hypothesis are significant effects in decay channels $X_{P S} \rightarrow \gamma l^{+} l^{-}, X_{P S} \rightarrow l^{+} l^{-} l^{+} l^{-}(l=e, \mu)$.
\end{abstract}

\section{Introduction}

In experiments [1] indications for excesses in the production of boson pairs $W W, W Z, Z Z$ were observed at invariant mass $M_{R} \simeq 2 \mathrm{TeV}$. Data for these processes are also present in works [2, 3]. Despite the fact that the wouldbe effect is not finally established yet, the publication causes numerous proposals for an interpretation mostly in terms of theories beyond the Standard Model (see, e.g. [4]).

In work [5] we have considered interpretation of the effect in terms of a weak isotopic spin 2 scalar $W W$ state. Indeed, pair of triplets $W^{a}$ could form a resonance state, the so-called $W$-ball. Of course the well known gauge interaction of these bosons with coupling $g\left(M_{W}\right)=0.65$ can not bind them in the resonance state with mass being of a $\mathrm{TeV}$ scale. However, there might exist also an additional effective interaction, e.g. the anomalous triple boson interaction $[7,8]$, which increases with increasing energy scale. In case the interaction becomes sufficiently strong at a $\mathrm{TeV}$ scale, it might lead to a formation of a resonance under discussion. Note, that the most recent data at $\sqrt{s}=13 \mathrm{TeV}$ [6] do not contradict estimates of [5].

There are recent data on $\gamma \gamma$ anomaly at $M(\gamma \gamma) \simeq 750 \mathrm{GeV}[11,12]$, which also caused numerous proposals for an interpretation [13]. Could this anomaly also be prescribed to analogous states? We would consider this problem in the present talk.

\footnotetext{
^e-mail: arbuzov@theory.sinp.msu.ru

$\star \star$ e-mail: zaytsev_iv@mail.ru
} 


\section{A model for the $W W$ resonance}

Now let us consider a possibility of a heavy resonance in case of an existence of the anomalous three-boson interaction, which in conventional notations [7, 8] looks like

$$
\begin{aligned}
& -\frac{G}{3 !} F \epsilon_{a b c} W_{\mu \nu}^{a} W_{\nu \rho}^{b} W_{\rho \mu}^{c} ; \quad G=-\frac{g \lambda}{M_{W}^{2}} \\
& W_{\mu \nu}^{a}=\partial_{\mu} W_{v}^{a}-\partial_{\nu} W_{\mu}^{a}+g \epsilon_{a b c} W_{\mu}^{b} W_{v}^{c} ;
\end{aligned}
$$

where $g \simeq 0.65$ is the electro-weak coupling. The best limitations for parameter $\lambda$ read [14]

$$
\lambda_{\gamma}=-0.022 \pm 0.019 ; \quad \lambda_{Z}=-0.09 \pm 0.06 ;
$$

where a subscript denote a neutral boson being involved in the experimental definition of $\lambda$. Let us emphasize that $F \equiv F\left(p_{i}\right)$ in definition (1) denotes a form-factor, which is either postulated as in original works $[7,8]$ or it is just uniquely defined as in works on a spontaneous generation of effective interaction (1) [9, 10]. In any case the form-factor guarantees the effective interaction to act in a limited region of the momentum space. That is it vanishes for momenta exceeding scale $\Lambda$. Formfactor $F$ is explicitly presented e.g. in work [5]. Calculations were done in the framework of an approximate scheme, which accuracy was estimated to be $\simeq(10-15) \%$ [15]. Would-be existence of effective interaction (1) leads to important non-perturbative effects in the electro-weak interaction.

In particular, one might expect resonances to appear in the system of two $W^{a}$-bosons. A possibility of an appearance of such states (W-balls) was already discussed, e.g. in works $[10,16]$. In the previous work [5] we studied the $2000 \mathrm{GeV}$ anomaly and appeared to the conclusion, that data can be described in terms of weak isospin 2 scalar resonance. The effect is due to anomalous interaction (1) and we come to a conclusion [5], that there is a possibility to describe data [1] with

$$
\lambda=-0.015 \pm 0.005 \text {. }
$$

There are recent indications for existence of the other effect: the $\gamma \gamma$ enhancement at invariant mass $M_{\gamma \gamma} \simeq 750 \mathrm{GeV}$. Let us consider this effect being explained by existence of zero weak isotopic spin pseudoscalar state $X_{P S}$, which interaction with electroweak bosons is described by the following expression

$$
L_{e f f}=\frac{G_{P S}}{4} \delta_{a b} \epsilon^{\mu \nu \rho \sigma} W_{\mu \nu}^{a} W_{\rho \sigma}^{b} X_{P S}
$$

Let us consider a Bethe-Salpeter equation for a pseudoscalar resonance consisting of two $W$. We consider such state corresponding to value of the weak isospin: $I=0$. With interaction (1) we have the following Bethe-Salpeter equation for state $X_{P S}$ in correspondence to diagrams presented in Fig 1 under assumption of existence of interaction (4).

$$
\begin{aligned}
& \Psi_{P S}=G_{P S}+\frac{G^{2}}{8 \pi^{2}}\left(\frac{1}{6 x} \int_{0}^{x} \Psi_{P S}(y) y^{2} d y-\frac{1}{2} \int_{0}^{x} \Psi_{P S}(y) y d y-\right. \\
& \left.\frac{x}{2} \int_{x}^{\infty} \Psi_{P S}(y) d y+\frac{x^{2}}{6} \int_{x}^{\infty} \frac{\Psi_{P S}(y)}{y} d y\right) ;
\end{aligned}
$$

where coupling constant $G_{P S}$ is defined by (4). Here in view of large value $M_{X} \simeq 0.75 \mathrm{TeV}$ of the wouldbe resonance we neglect $W$ mass.

$$
z=\frac{G^{2} x^{2}}{128 \pi^{2}} ; t=\frac{G^{2} y^{2}}{128 \pi^{2}}
$$




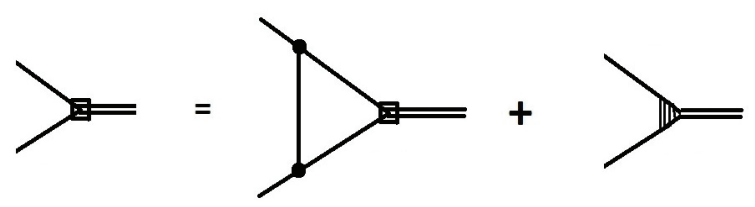

Figure 1. Diagram form of equation (5). Simple lines represent $W$-s, a double line corresponds to the resonance, black circles correspond to interaction (1), squares correspond to BS wave function. The triangle corresponds to vertex (4).

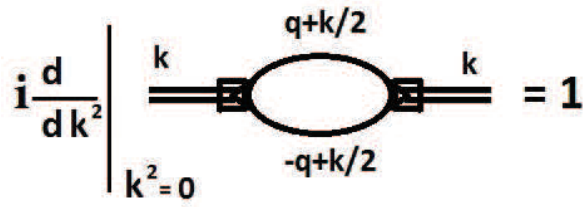

Figure 2. Diagram representation of normalization condition for coupling $G_{P S}$. Simple lines represent $W$-s, a double line corresponds to the resonance, squares correspond to BS wave function.

we come to the following equation

$$
\begin{aligned}
& \Psi_{P S}(z)=G_{P S}+\frac{4}{3 \sqrt{z}} \int_{0}^{z} \Psi_{R}(t) \sqrt{t} d t-4 \int_{0}^{z} \Psi_{P S}(t) d t- \\
& 4 \sqrt{z} \int_{z}^{\infty} \frac{\Psi_{P S}(t)}{\sqrt{t}} d t+\frac{4 z}{3} \int_{z}^{\infty} \frac{\Psi_{P S}(t)}{t} d t .
\end{aligned}
$$

Equation (7) satisfies condition

$$
\Psi_{P S}(0)=G_{P S}
$$

By successive differentiations of equation (7) we obtain a Meijer differential equation for function $\Psi_{P S}(z)$

$$
\left[\left(z \frac{d}{d z}+\frac{1}{2}\right)\left(z \frac{d}{d z}\right)\left(z \frac{d}{d z}-\frac{1}{2}\right)\left(z \frac{d}{d z}-1\right)+z\right] \Psi_{P S}(z)=0 .
$$

Then the solution, which fulfill boundary condition both at zero and at the infinity (see e.g. [18]) is the following

$$
\Psi_{P S}(z)=\frac{G_{P S}}{2} G_{04}^{30}\left(\left.z\right|_{0,1 / 2,1,-1 / 2}\right) .
$$


The normalization condition for Bethe-Salpeter wave function (10) give according to diagram Fig. 2 with account of definition (6) the following relation

$$
\begin{aligned}
& \frac{9}{64 \pi^{2}} \int_{0}^{\infty} d y \Psi_{P S}(y)^{2}=\frac{9 \sqrt{2} G_{P S}^{2}}{16 \pi G} I=1 ; \\
& I=\int_{0}^{\infty} \frac{G_{04}^{30}\left(\left.t\right|_{0,1 / 2,1,-1 / 2}\right)^{2}}{2 \sqrt{t}} d t=\frac{\pi}{8} .
\end{aligned}
$$

With values $I(11), g=0.65$ and with account of (1) we obtain coupling $G_{P S}$

$$
G_{P S}=\frac{8}{3 M_{W}} \sqrt{g|\lambda| \sqrt{2}}=0.00318 \frac{1}{G e V}
$$

where numerical value corresponds to $\lambda=\lambda_{0}=-0.01$, that is safely inside restrictions (2) and estimates (3).Value (12) corresponds to scale $\Lambda \simeq 0$. We take value (12) for estimates of effects, bearing in mind, that for other values of scale $\Lambda$ coupling $G_{P S}$ is defined by solution (10), namely

$$
G_{P S}(\Lambda)=\frac{G_{P S}}{2} G_{04}^{30}\left(\left.z_{\Lambda}\right|_{0,1 / 2,1,-1 / 2}\right) ; z_{\Lambda}=\frac{G^{2} \Lambda^{4}}{128 \pi^{2}} .
$$

We evaluate the pseudoscalar resonance decay probabilities with $\Lambda_{D}=M_{P S}=750 \mathrm{GeV}$. For estimations of cross sections we take $\Lambda$ in correspondence to maxima of structure functions. That is $\Lambda(\sqrt{s}) \simeq \sqrt{s} / 7$. Then for the decay and for two values $\sqrt{s}=8 \mathrm{TeV}$ and $\sqrt{s}=13 \mathrm{TeV}$ we have the following values for effective coupling $G_{P S}$

$$
\begin{aligned}
& G_{P S}\left(\Lambda_{D}\right)=G_{P S}(0.75 T e V)=0.00303 ; \\
& G_{P S}(8 T e V / 7)=0.00285 ; G_{P S}(13 T e V / 7)=0.00242 .
\end{aligned}
$$

Thus we have interaction (4) with parameters $G_{P S}(12,14)$ and $M_{P S}=750 \mathrm{GeV}$. We use well-known relation

$$
W^{0}=\cos \theta_{W} Z+\sin \theta_{W} A ;
$$

and obtain for partial decay widths of the pseudoscalar $X_{P S}$

$$
\begin{aligned}
& \Gamma\left(W^{+} W^{-}\right)=31.06 \mathrm{GeV}(43.5 \%) ; \quad \Gamma(Z Z)=9.01 \mathrm{GeV}(12.6 \%) ; \\
& \Gamma(Z \gamma)=4.72 \mathrm{GeV}(6.6 \%) ; \quad \Gamma(\gamma \gamma)=0.91 \mathrm{GeV}(1.2 \%) ; \\
& \left.\Gamma\left(W^{+} W^{-} Z\right)=21.34 \mathrm{GeV} 29.9 \%\right) ; \quad \Gamma\left(W^{+} W^{-} \gamma\right)=4.45 \mathrm{GeV}(6.2 \%) \\
& \Gamma_{t}\left(X_{P S}\right)=71.49 \mathrm{GeV} .
\end{aligned}
$$

We would present also probabilities for the following specific channels, where $l$ means light lepton $(\mu, e)$

$$
\Gamma\left(l^{+} l^{-} \gamma\right)=0.404 \mathrm{GeV}(0.64 \%) ; \Gamma\left(l^{+} l^{-} l^{+} l^{-}\right)=0.0411 \mathrm{GeV}(0.065 \%)
$$

Then we calculate cross sections for $X_{P S}$ production in $p p$ collisions for $\sqrt{s}=8 \mathrm{TeV}$ and for $\sqrt{s}=$ $13 \mathrm{TeV}$. In doing this we use CompHEP package [19].

Thus we consider possible pseudoscalar neutral resonance with mass $\approx 750 \mathrm{TeV}$, which decay into

$$
W^{+} W^{-} ; \quad Z Z ; \quad Z \gamma, \quad \gamma \gamma
$$


Table 1. Results for cross-sections of $X_{P S}$ production in $p+p$ collisions at $\sqrt{s}=8 \mathrm{TeV}$ and $\sqrt{s}=13 \mathrm{TeV}$ with $\lambda=-0.01\left(G_{P S}=0.00318 \mathrm{GeV}^{-1}\right)$.

\begin{tabular}{lcc}
\hline$p+p \rightarrow X+$ & $\sigma f b, 8 \mathrm{TeV}$ & $\sigma \mathrm{fb}, 13 \mathrm{TeV}$ \\
\hline$W^{+} X_{P S}$ & 12.96 & 23.10 \\
\hline$W^{-} X_{P S}$ & 3.70 & 7.89 \\
\hline$Z X_{P S}$ & 5.87 & 11.31 \\
\hline$\gamma X_{P S}$ & 1.85 & 3.54 \\
\hline$q(\bar{q})$ jets $X_{P S}$ & 81.68 & 131.10 \\
\hline$W^{+} W^{-} X_{P S}$ & 36.70 & 289.87 \\
\hline$W^{+} Z X_{P S}$ & 27.36 & 208.05 \\
\hline$W^{-} Z X_{P S}$ & 6.60 & 53.45 \\
\hline$W^{+} \gamma X_{P S}$ & 1.61 & 4.47 \\
\hline$W^{-} \gamma X_{P S}$ & 0.40 & 1.10 \\
\hline$\sigma\left(X_{P S}\right)_{t o t}$ & 178.74 & 734.11 \\
\hline
\end{tabular}

According to Table 1 the cross-section of the resonance production at $\sqrt{s}=8 \mathrm{TeV}$ is four times less than at $\sqrt{s}=13 \mathrm{TeV}$. Available $\sqrt{s}=8 \mathrm{TeV}$ data [20-22] do not contradict to our estimates with account of branching ratios (16). Namely, for $\sqrt{s}=8 \mathrm{TeV}$ we have

$$
\sigma\left(p p \rightarrow X_{P S}\right) \cdot B R\left(X_{P S} \rightarrow \gamma \gamma\right)=178.8 \cdot 0.012=2.15 f b
$$

that do not contradict the most recent limitations [23]. Limitations for $W W$ and $Z Z$ decay modes [24, 25] also do not contradict the present results. For example, CMS data [22] give for $750 \mathrm{GeV}$ resonance with width $\simeq 100 \mathrm{GeV}$ limitation $\sigma B R\left(X_{P S} \rightarrow \gamma \gamma\right)<40 \mathrm{fb}$ with prediction $5.2 \mathrm{fb}$ from Table 1. Let us note, that our result for effect in channel $X_{P S} \rightarrow Z Z$ gives for $l^{+} l^{-}(l=e, \mu)$ decays of $Z$-bosons with integral luminosity $L=5.3 \mathrm{fb}^{-1}[20]$ the following estimate for an event number

$$
\begin{aligned}
& \sigma\left(X_{P S}, 8 T e V\right) \cdot B R\left(X_{P S} \rightarrow l^{+} l^{-} l^{+} l^{-}\right) \cdot L= \\
& 178.74 \cdot 0.00065 \cdot 5.3=0.62 .
\end{aligned}
$$

It is worth mentioning, that in experimental results at $\sqrt{s}=8 \mathrm{TeV}$ [20] there is one event just at $M\left(l^{+} l^{-} l^{+} l^{-}\right)=750 \mathrm{GeV}$ and no other events for $M\left(l^{+} l^{-} l^{+} l^{-}\right)>600 \mathrm{GeV}$. Of course this coincidence proves nothing due to the poor statistics, we may only state, that results [20] do not contradict our estimates.

Now what for $\sqrt{s}=13 \mathrm{TeV}$ ? First of all let us estimate an effect in channel $\gamma \gamma$. We have for possible number of events with (16) and data from Table 1

$$
N_{\gamma \gamma}=\sigma\left(p p \rightarrow X_{P S}\right) \cdot B R\left(X_{P S} \rightarrow \gamma \gamma\right) \cdot L=8.81 \cdot L\left(f b^{-1}\right) .
$$

Thus we have for $L \simeq 3 \mathrm{fb}^{-1}$ few tens events, that agrees observations [11, 12].

It may be advisable to study effect not only in channel $X_{P S} \rightarrow \gamma \gamma$ but also in channel $X_{P S} \rightarrow$ $\gamma l^{+} l^{-}$. According to (16) we have

$$
\frac{B R\left(X_{P S} \rightarrow \gamma l^{+} l^{-}\right)}{B R\left(X_{P S} \rightarrow \gamma \gamma\right)}=\frac{0.445}{1.0}=0.445
$$


that is actually only two times smaller than already observed effect in $2 \gamma$.

Let us also calculate the effect for $l^{+} l^{-} l^{+} l^{-}$at $\sqrt{s}=13 \mathrm{GeV}$ in the resonance region $\simeq 750 \mathrm{GeV}$

$$
N\left(l^{+} l^{-} l^{+} l^{-}\right)=\sigma\left(X_{P S}, 13 T e V\right) \cdot B R\left(X_{P S} \rightarrow l^{+} l^{-} l^{+} l^{-}\right)=0.48 L\left(f b^{-1}\right) .
$$

So for $L \sim 20 \mathrm{fb}^{-1}$ the effect in four leptons channel may become noticeable. The more so as for this channel background conditions are favorable [20]. Effects $X_{P S} \rightarrow \gamma l^{+} l^{-} ; X_{P S} \rightarrow l^{+} l^{-} l^{+} l^{-}$ with intensities $(23,24)$ would confirm definitely the interpretation of the $750 \mathrm{GeV}$ state being W-ball. Note, that existing limitations on a possible extra contribution of decay $X_{P S} \rightarrow \gamma Z$ with invisible decay $Z \rightarrow \bar{v} v$ [26] do not contradict our estimates.

There is one more suspicious point in data by ATLAS [27], where process

$$
p+p \rightarrow j e t s+Z\left(\rightarrow l^{+} l^{-}\right)+\text {missing } E_{T} ;
$$

was studied at $\sqrt{s}=8 \mathrm{TeV}$. SM calculations for the number of events for integrated luminosity $L=20.3 \mathrm{fb}^{-1}$ give $N=9.8 \pm 2.9 \pm 1.4$. Experiment [27] gives $N=29$ events.

However with our premises we have for process $p+p \rightarrow X_{P S}+$ everything with decay $X_{P S} \rightarrow$ $Z\left(\rightarrow l^{+} l^{-}\right)+Z(\rightarrow \bar{v} v)$ also missing $E_{T}$ due to the invisible $Z$-decay. Thus we have for the additional contribution

$$
\Delta N=\sigma\left(p p \rightarrow X_{P S}\right) \cdot L \cdot B R\left(X_{P S} \rightarrow Z Z\right) \cdot 2 \cdot B R\left(Z \rightarrow l^{+} l^{-}\right) B R(Z \rightarrow \bar{v} v)=12.3
$$

for results in tables. Contribution (26) corresponds to the discrepancy within error bars. In any case the data do not contradict the $W$-ball interpretation of $X(750)$.

Let us remind, that all the estimates were made with $\lambda=-0.01$. Calculations for another value of $\lambda$ are straightforward with the prescriptions presented above.

\section{Conclusion}

Existence of W-balls would testify for anomalous gauge interaction (1), which would be due to nonperturbative effects in the electroweak interaction. Thus we could come to important conclusion, that non-perturbative contributions are appropriate not only to $\mathrm{QCD}$, but to the electroweak interaction as well. In this case the anomalies in the electroweak boson pair production do not contradict the Standard Model and do not need extra efforts for a choice of a theory beyond the SM.

Data on effects under discussion might give information on a value of parameter $\lambda$. According to our considerations it could be expected in range $\lambda=-0.010 \pm 0.005$. Of course experiments on direct measurement of $\lambda$, e.g. in processes of $W^{+} W^{-}, W^{ \pm} Z(\gamma)$ production are also quite desirable.

\section{Acknowledgments}

The work is supported in part by grant NSh-7989.2016.2 of the President of Russian Federation.

\section{References}

[1] G. Aad et.al. (ATLAS Collaboration), J. High Energy Phys., 1512, 055 (2015).

[2] V. Khachatryan et.al. (CMS Collaboration), J. High Energy Phys., 1408, 173 (2014).

[3] V. Khachatryan et.al. (CMS Collaboration), J High Energy Phys., 1408, 174 (2014).

[4] D. Gonsalves, F. Krauss and M. Spannovsky, Phys. Rev. D 92 (2015) Article 053010. 
[5] B. A. Arbuzov and I. V. Zaitsev, Int. J. Mod. Phys. A 30 (2015) Article 1550221.

[6] G. Aad et.al. (ATLAS Collaboration), arXiv: 1606.04833 [hep-ex] (2016).

[7] K. Hagiwara, R.D. Peccei, D. Zeppenfeld and K. Hikasa, Nucl. Phys. B 282, 253 (1987).

[8] K. Hagiwara, S. Ishihara, R. Szalapski and D. Zeppenfeld, Phys. Rev. D 48, 2182 (1993).

[9] B. A. Arbuzov, Eur. Phys. J. C 61, 51 (2009).

[10] B. A. Arbuzov and I. V. Zaitsev, Phys. Rev. D 85 (2012) Article 093001.

[11] G. Aad et.al. (ATLAS Collaboration), arXiv: 1606.03833 [hep-ex] (2016).

[12] V. Khachatryan et al. (CMS Collaboration), arXiv: 1606.04093 [hep-ex] (2016).

[13] J. Ellis et al., arXiv: 1512.05327 [hep-ph].

[14] K. A. Olive et al. (Particle Data Group), Review of particle physics, Chin. Phys. C 38 (2014) Article 090001.

[15] B. A. Arbuzov, Theor. Math. Phys. 140, 1205 (2004).

[16] B. A. Arbuzov and I. V. Zaitsev, Int. J. Mod. Phys. A 27 (2012) Article 1250012.

[17] H. Bateman and A. Erdélyi, Higher Transcendental Functions V.1, McGraw-Hill, New-York, Toronto, London, 1953.

[18] B. A. Arbuzov, Non-perturbative Effective Interactions in the Standard Model, De Gruyter, Berlin, 2014.

[19] E. E. Boos et al. (CompHEP Collaboration), Nucl. Instr. Meth. A 534, 250 (2004).

[20] V. Khachatryan et al. (CMS Collaboration), Phys. Lett. B 721, 190 (2013).

[21] G. Aad et al. (ATLAS Collaboration), Phys. Rev. Lett 113 (2015) Article 71801.

[22] V. Khachatryan et al. (CMS Collaboration), Phys. Lett. B 750, 494 (2015).

[23] G. Aad et al. (ATLAS Collaboration), Phys. Rev. D 92 (2015) Article 032004.

[24] G. Aad et al. (ATLAS Collaboration), Eur. Phys. J. C, 75, 209 (2015).

[25] G. Aad et al. (ATLAS Collaboration), arXiv: 1512.05099 [hep-ex].

[26] V. Khachatryan et al. (CMS Collaboration), Phys. Lett. B 755, 102 (2016).

[27] G. Aad et al. (ATLAS Collaboration), Eur. Phys. J. C 75, 318 (2015). 